\title{
COMPUTATIONAL INVESTIGATION OF POISSON'S RATIO AND ITS RELATIONSHIP TO CRYSTAL STRUCTURE
}

\author{
John Dagdelen \\ John Dagdelen, Department of Materials Science and Engineering, UC Berkeley \\ jdagdelen@berkeley.edu
}

A material's Poisson's ratio describes the magnitude of transverse strain that results when it undergoes a tensile strain. However, Poisson's ratio can be generalized in anisotropic crystals though the elastic tensor to give the single-direction Poisson's ratio for a strain in any specific crystallographic direction. In a few rare cases, a crystalline material can possess extreme directional Poisson's ratios and exhibit surprising properties as a result. One example of this phenomenon is the appearance of an overall negative average Poisson's ratio in the polycrystalline bulk. Such materials have been shown to have exceptional mechanical properties and are enabling advancements in technologies like high-precision sensors, tougher ceramics, and impact-resistant composites. While only a few hundred experimental elastic tensors have been measured to date, new computational methods are now enabling researchers to calculate the elastic tensors of thousands of materials at a time. In this talk (or poster), I discuss how we can use high-throughput computational screening methods based on new descriptors for similarity between crystal structures in combination with open materials databases like the Materials Project database to identify materials that are likely to exhibit unusual mechanical properties (such as negative Poisson's ratio). I then show how the mechanical properties of these materials can be further investigated through computation of the elastic tensor via density functional theory calculations to yield a complete topographical picture of Poisson's ratio for a given crystalline material. 